\title{
Intrinsic dissolution simulation of highly and poorly soluble drugs for BCS solubility classification
}

\author{
Marcelo Dutra Duque ${ }^{1,2, *}$, Michele Georges Issa ${ }^{1}$, Daniela Amaral Silva ${ }^{1}$, Beatriz Ayumi Sakamoto \\ Kakuda², Leticia Norma Carpentieri Rodrigues ${ }^{2}$, Raimar Löbenberg ${ }^{3}$, Humberto Gomes Ferraz ${ }^{1}$ \\ ${ }^{1}$ Department of Pharmacy, Faculty of Pharmaceutical Sciences, Universidade de São Paulo, Brazil \\ ${ }^{2}$ Department of Exact and Earth Sciences, Institute of Environmental, Chemical and Pharmaceutical Sciences, \\ Universidade Federal de São Paulo - UNIFESP, Brazil \\ ${ }^{3}$ Faculty of Pharmacy \& Pharmaceutical Science, Centre for Pharmacy \& Health Research, University of Alberta, Canada
}

\section{ABSTRACT}

e-mail: marceloduque@outlook.com

Intrinsic dissolution testing allows characterizing drug substances through its dissolution rate when exposed to a specified surface area in a specific dissolution media. This can be used to determine if a drug substance is highly or poorly soluble. In this work, DDDPlus version 4.0 (Simulations Plus, Inc.) was used to simulate intrinsic dissolution experiments for pyrimethamine and metronidazole. These drugs have low and high solubility properties. Predicted intrinsic dissolution rates (IDR) were compared to observed in vitro IDR. Physicochemical parameters from literature and the experimental conditions of the intrinsic dissolution tests for each drug were used as input data into the software. The program was able to predict the IDR of pyrimethamine and metronidazole within a $\mathrm{pH}$ range of 1.0 to 7.2. Observed and predicted IDR values for both drugs showed high correlations $\left(R^{2}>0.9424\right)$. The IDR values from simulations showed the $\mathrm{pH}$-dependent solubility of pyrimethamine and metronidazole, allowing us to classify the solubility according to the Biopharmaceutics Classification System (BCS). Intrinsic dissolution test simulations using DDDPlus can be used to obtain a BCS solubility classification of a drug substance, helping to reduce the number of laboratory experiments.

KEYWORDS: pyrimethamine; metronidazole; intrinsic dissolution rate; BCS; DDDPlus; dissolution

\section{INTRODUCTION}

ntrinsic dissolution is a characterization test for active pharmaceutical ingredients (APIs) where the dissolution rate of a drug is determined from a specific surface area exposed to a dissolution medium at certain rotation speed. $(1,2)$ The intrinsic dissolution rate (IDR) can be used to estimate the solubility class of a substance according to the Biopharmaceutics Classification System (BCS) guidelines. $(3,4)$ It can be also valuable to evaluate differences between polymorphs and solvates. (2)

According to the BCS, drugs are classified as high or low solubility. (5) Equilibrium solubility testing (shake flask method) is recommended by the US Food and Drug Administration to determine the solubility of drugs, allowing to obtain their BCS class. (6-10) However, this method requires the saturation of aqueous solutions, which can be a challenge depending on the drug characteristics, mainly in the early stages of development. For this purpose, intrinsic dissolution is an important characterization test that can be used to obtain the solubility of drugs according to BCS guidelines. $(3,4)$

*Corresponding author
The dissolution of an API in a formulation is affected by different factors such as the test conditions (temperature, rotation speed, $\mathrm{pH}$, nature of dissolution medium) and formulation factors, such as compaction pressure and excipient interactions. (1) Using design of experiments (DOE) is one way to evaluate the most appropriate test conditions. $(11,12)$

Although only a few milligrams of API is used in intrinsic dissolution testing, developing a method requires a reasonable number of experiments to evaluate the impact of the test conditions on the IDR. One of the strategies to reduce the number of experiments is using fractional factorial design to develop appropriate intrinsic dissolution methods. $(11,12)$ However, depending on the quantity of the available sample, this step can be unfeasible, in early stages of the development process, where only limited material is available. Approaches, which can further reduce experimental testing, are highly desirable.

DDDPlus $^{\mathrm{TM}}$ (Dose Disintegration and Dissolution) version 4.0, designed by Simulations Plus Inc., is a computer program used to simulate in vitro dissolution tests 
employing USP apparatuses 1 (basket), 2 (paddle), and 4 (flow-through cell) and intrinsic dissolution using the rotating disk method. (13) The use of DDDPlus to simulate in vitro dissolution from tablets containing low soluble drugs, montelukast sodium and glyburide, was previously demonstrated. (14) This software can simulate intrinsic dissolution tests, saving time, reducing the number of experiments to investigate suitable IDR conditions.

The objective of this work was to demonstrate the use of the computer program DDDPlus as a tool for BCS solubility classification by simulating intrinsic dissolution tests for the poorly soluble drug, pyrimethamine, and the highly soluble drug, metronidazole.

\section{MATERIALS AND METHODS \\ Materials}

Pyrimethamine and metronidazole were kindly donated by Farmanguinhos Laboratory/Fiocruz (Rio de Janeiro, Brazil) and Micro Service Micronização e Processos (Diadema, Brazil), respectively. Both APIs were of pharmaceutical grade and were used as received. Hydrochloric acid $(\mathrm{HCl})$ P.A. 37\%, glacial acetic acid, sodium acetate, potassium phosphate monobasic monohydrate, potassium chloride, and sodium hydroxide were purchased from Labsynth Products Laboratories (Diadema, Brazil) and used to prepare the buffer solutions as described below.

Hydrochloric acid pH 1.2 was prepared by accurately pipetting $7 \mathrm{~mL}$ of $\mathrm{HCl}$ P.A. $37 \%$ and weighing $3.73 \mathrm{~g}$ of potassium chloride per liter. Hydrochloric acid $0.1 \mathrm{M}$ was prepared by pipetting $8.3 \mathrm{~mL}$ of HCl P.A. $37 \%$ and adding purified water to $1 \mathrm{~L}$.

Acetate buffer $\mathrm{pH} 4.5$ was prepared by dissolving $2.99 \mathrm{~g}$ of sodium acetate, $14 \mathrm{~mL} 2-\mathrm{N}$ acetic acid solution, and purified water. Phosphate buffer pH 7.2 was prepared by dissolving $6.8 \mathrm{~g}$ of potassium phosphate monobasic monohydrate and $1.4 \mathrm{~g}$ of $\mathrm{NaOH}$, and adding purified water. The amounts of material were used to prepare $1 \mathrm{~L}$ of buffer solutions.

\section{Intrinsic dissolution tests}

For pyrimethamine, intrinsic dissolution testing was performed according to a fractional factorial design $3^{3-1}$ using Statistica ${ }^{\circledR} 12.0$ (StatSoft, Inc., Tulsa, OK, USA) including factors such as compaction pressure $(1.75,3.5$, and $7.0 \mathrm{kN})$, nature of the dissolution medium $(\mathrm{HCl} \mathrm{pH} \mathrm{1.2;}$ acetate buffer $\mathrm{pH} 4.5$, and phosphate buffer $\mathrm{pH}$ 7.2), and rotation speed $(50,100$, and $200 \mathrm{rpm})$, generating the experiments described in Table 1.

Table 1. Pyrimethamine Intrinsic Dissolution Test Conditions
\begin{tabular}{|c|c|c|c|c|}
\hline $\begin{array}{c}\text { Standard } \\
\text { order }\end{array}$ & $\begin{array}{c}\text { Run } \\
\text { order }\end{array}$ & $\begin{array}{c}\text { Compaction } \\
\text { pressure } \\
(\mathrm{kN})\end{array}$ & Dissolution media & $\begin{array}{c}\text { Rotation } \\
\text { speed (rpm) }\end{array}$ \\
\hline 1 & P1 & 1.75 & $\mathrm{HCl} \mathrm{pH} \mathrm{1.2}$ & 50 \\
\hline 2 & P4 & 1.75 & $\begin{array}{c}\text { Acetate buffer pH } \\
4.5\end{array}$ & 200 \\
\hline 3 & P7 & 1.75 & $\begin{array}{c}\text { Phosphate buffer } \\
\text { pH 7.2 }\end{array}$ & 100 \\
\hline 4 & P2 & 3.5 & $\begin{array}{c}\mathrm{HCl} \mathrm{pH} 1.2 \\
4.5\end{array}$ & 200 \\
\hline 5 & P5 & 3.5 & $\begin{array}{c}\text { Acetate buffer pH } \\
4.5\end{array}$ & 100 \\
\hline 6 & P8 & 3.5 & $\begin{array}{c}\text { Phosphate buffer } \\
\text { pH 7.2 }\end{array}$ & 50 \\
\hline 7 & P3 & 7.0 & HCl pH 1.2 & 100 \\
\hline 8 & P6 & 7.0 & $\begin{array}{c}\text { Acetate buffer pH } \\
4.5\end{array}$ & 50 \\
\hline 9 & P9 & 7.0 & $\begin{array}{c}\text { Phosphate buffer } \\
\text { pH 7.2 }\end{array}$ & 200 \\
\hline
\end{tabular}

$\mathrm{HCl}$, hydrochloric acid.

Rotating disk apparatuses from Varian Inc. (Palo Alto, CA, USA) coupled to a D-800 Logan dissolution tester (Logan Instruments Corp., NJ, USA) was used to perform the intrinsic dissolution tests. About $150 \mathrm{mg}$ of the drug was weighed in triplicate and compacted using a hydraulic press (American Lab., São Paulo, Brazil). The temperature used was $37 \pm 0.5^{\circ} \mathrm{C}$; the volume of the dissolution medium was $900 \mathrm{~mL}$, and aliquots of $5 \mathrm{~mL}$ were collected in intervals until a sufficient number of points were obtained.

Each aliquot of dissolution medium was immediately replaced at the same volume and temperature. The amount of drug dissolved was analyzed by a spectrophotometric method in a UV-VIS Cary 50 (Varian Inc.) using quartz cuvettes of $10.0 \mathrm{~mm}$ at $273 \mathrm{~nm}$, using each dissolution media as blank.

The visual evaluation of the compacted drug's surface was monitored during the experiments to avoid erroneous data from possible alterations, which can be detected by the presence of curvatures in the plots and low values of linearity. All dissolution media were previously degassed to prevent bubble formation, which could hamper the dissolution of the drug. $(1,3)$

Intrinsic dissolution rate was calculated according to United States Pharmacopeia (1). The amount of drug dissolved (mg) was plotted versus time (s), and through linear regression, $\mathrm{R}^{2}$ and the corresponding equation was obtained. The slope of this equation is the dissolution rate, and this value was divided by the exposed surface area $\left(0.5 \mathrm{~cm}^{2}\right)$ to obtain the IDR $\left(\mathrm{mg} / \mathrm{s} / \mathrm{cm}^{2}\right)$. 
For metronidazole, the results from an earlier study (11) were used for the simulations. The authors conducted the experiments according to a $3^{4-1}$ fractional factorial design, resulting in 27 experiments, including factors such as compaction pressure, rotation speed, dissolution media, and metronidazole micronization degree. The study confirmed that API micronization did not influence IDR results. (11)

Therefore, in this work, we considered only compaction pressure, rotation speed, and dissolution media as parameters, since there is no influence by particle size on IDR. Furthermore, DDDPlus disables the use of particle size distribution of the drug when intrinsic dissolution is selected. It resulted in a $3^{3-1}$ fractional factorial design (Table 2), from which corresponding in vitro IDR values were used for comparison.

Table 2. Metronidazole Intrinsic Dissolution Test Conditions

\begin{tabular}{|c|c|c|c|c|}
\hline $\begin{array}{c}\text { Standard } \\
\text { order }\end{array}$ & $\begin{array}{c}\text { Run } \\
\text { order }\end{array}$ & $\begin{array}{c}\text { Compaction } \\
\text { pressure } \\
(\mathbf{k N})\end{array}$ & Dissolution media & $\begin{array}{c}\text { Rotation } \\
\text { speed (rpm) }\end{array}$ \\
\hline 1 & M1 & 3.5 & HCl 0.1 M & 50 \\
\hline 2 & M8 & 3.5 & Purified water & 100 \\
\hline 3 & M6 & 3.5 & $\begin{array}{c}\text { Phosphate buffer } \\
\text { pH 7.2 }\end{array}$ & 75 \\
\hline 4 & M16 & 7.0 & HCl 0.1 M & 100 \\
\hline 5 & M14 & 7.0 & Purified water & 75 \\
\hline 6 & M12 & 7.0 & $\begin{array}{c}\text { Phosphate buffer } \\
\text { pH 7.2 }\end{array}$ & 50 \\
\hline 7 & M22 & 10.5 & HCl 0.1 M & 75 \\
\hline 8 & M20 & 10.5 & Purified water & 50 \\
\hline 9 & M27 & 10.5 & $\begin{array}{c}\text { Phosphate buffer } \\
\text { pH 7.2 }\end{array}$ & 100 \\
\hline
\end{tabular}

$\mathrm{HCl}$, hydrochloric acid.

\section{Computer simulations using DDDPlus}

DDDPlus software was used to simulate the intrinsic dissolution tests. A database for pyrimethamine and one for metronidazole were created in the software.

The ADMET Predictor ${ }^{\mathrm{TM}}$ module present in the computer program, GastroPlus ${ }^{\mathrm{TM}}$ (Simulations Plus Inc.) can accurately predict absorption, metabolism, elimination, and toxicity characteristics of substances from its molecular structure. This module was used to estimate solubility vs $\mathrm{pH}$ data for each drug at $25^{\circ} \mathrm{C}$. For this purpose, files containing the molecular structure of each drug were used in this module, and the following solubility data were obtained: pyrimethamine solubility $=0.03 \mathrm{mg}$ /
$\mathrm{mL}$ at $\mathrm{pH} 8.37$ and metronidazole solubility $=13.42 \mathrm{mg} /$ $\mathrm{mL}$ at $\mathrm{pH}$ 7.66. Although the estimated solubility was at $25^{\circ} \mathrm{C}$, these values were used as input data in DDDPlus because they are in accordance to shake-flask results experiments conducted at $37^{\circ} \mathrm{C}$ in our lab: pyrimethamine solubility $=0.04 \mathrm{mg} / \mathrm{mL}$ at phosphate buffer $0.05 \mathrm{M}$ $\mathrm{pH} 7.5$ and metronidazole solubility $=13.14 \mathrm{mg} / \mathrm{mL}$ at phosphate buffer $0.05 \mathrm{M} \mathrm{pH}$ 7.2. The pKa values of 7.4 (pyrimethamine) and 2.55 (metronidazole) were obtained from the literature $(15,16)$; particle density $(1.2 \mathrm{~g} / \mathrm{mL})$, precipitation time $(900 \mathrm{~s})$, and diffusion coefficient $(0.5$ $\mathrm{cm}^{2} / \mathrm{s} \times 10^{-5}$ ) were used as default values from DDDPlus.

Intrinsic dissolution tests conditions for both pyrimethamine (Table 1) and metronidazole (Table 2) were used as input data into the Experimental tab of the software. Single simulations were performed for each experiment. As described in the pyrimethamine intrinsic dissolution tests section, IDR was calculated from the simulated data for both drugs. The values from simulated intrinsic dissolution tests were compared to the in vitro results.

\section{Statistical analysis}

Observed IDR values for pyrimethamine and metronidazole were analyzed using Statistica 12.0. Analysis of Variance (ANOVA) was used with $p<0.05$ to establish statistical significant differences; Pareto charts where generated for each API.

\section{RESULTS}

\section{Intrinsic dissolution and computer simulations}

For pyrimethamine, the amount of drug dissolved was plotted against time to obtain a slope to calculate the IDR. A simulated amount of pyrimethamine dissolved versus time from DDDPlus was used to predict IDR.

Observed and predicted IDR values for pyrimethamine and the regression coefficients are shown in Table 3.

Similarly, for metronidazole the simulated amounts dissolved versus time were used to predict the IDR, and in vitro IDR data were used to compare to the predicted IDR with the observed IDR (Table 4).

For both drugs, Pareto charts showed that only dissolution media presented a significant influence on IDR (Figures 1 and 2), which was also confirmed by ANOVA of IDR observed results with significant influence for the variable dissolution medium $(p=0.021$ for pyrimethamine and $p=$ 0.011 for metronidazole). 
Table 3. In Vitro (Observed) and In Silico (Predicted) Intrinsic Dissolution Rate (IDR) and $R^{2}$ Values for Pyrimethamine

\begin{tabular}{|c|c|c|c|c|}
\hline \multirow{2}{*}{ Experiment } & \multirow{2}{*}{$\begin{array}{c}\text { Dissolution } \\
\text { media }\end{array}$} & \multicolumn{2}{|c|}{ IDR $\left(\mathrm{mg} / \mathrm{s} / \mathrm{cm}^{2}\right)$} & $\begin{array}{c}\text { Observed } \\
\text { vs Predicted } \\
\text { IDR }\end{array}$ \\
\cline { 3 - 5 } & & Observed & Predicted & $\mathrm{R}^{2}$ \\
\hline P1 & $\mathrm{HCl} \mathrm{pH} \mathrm{1.2}$ & 0.0028 & 0.0030 & 0.9956 \\
\hline P2 & $\mathrm{HCl} \mathrm{pH} \mathrm{1.2}$ & 0.0052 & 0.0050 & 0.9987 \\
\hline P3 & $\mathrm{HCl} \mathrm{pH} \mathrm{1.2}$ & 0.0046 & 0.0046 & 0.9919 \\
\hline P4 & $\begin{array}{c}\text { Acetate buffer } \\
\text { pH 4.5 }\end{array}$ & 0.0068 & 0.0072 & 0.9976 \\
\hline P5 & $\begin{array}{c}\text { Acetate buffer } \\
\text { pH 4.5 }\end{array}$ & 0.0072 & 0.0070 & 0.9865 \\
\hline P6 & $\begin{array}{c}\text { Acetate buffer } \\
\text { pH 4.5 }\end{array}$ & 0.0080 & 0.0072 & 0.9860 \\
\hline P7 & $\begin{array}{c}\text { Phosphate } \\
\text { buffer pH 7.2 }\end{array}$ & 0.00008 & 0.00008 & 0.9762 \\
\hline P8 & $\begin{array}{c}\text { Phosphate } \\
\text { buffer pH 7.2 }\end{array}$ & 0.00016 & 0.00016 & 0.9881 \\
\hline P9 & $\begin{array}{c}\text { Phosphate } \\
\text { buffer pH 7.2 }\end{array}$ & 0.00008 & 0.00012 & 0.9424 \\
\hline
\end{tabular}

Table 4. In Vitro (Observed) and In Silico (Predicted) Intrinsic Dissolution Rate (IDR) and $R^{2}$ Values for Metronidazole

\begin{tabular}{|c|c|c|c|c|}
\hline \multirow{2}{*}{ Experiment } & \multirow{2}{*}{$\begin{array}{c}\text { Dissolution } \\
\text { media }\end{array}$} & \multicolumn{2}{|c|}{ IDR $\left(\mathrm{mg} / \mathrm{s} / \mathrm{cm}^{2}\right)$} & $\begin{array}{c}\text { Observed } \\
\text { vs Predicted } \\
\text { IDR }\end{array}$ \\
\cline { 3 - 5 } & & Observed & Predicted & $\mathbf{R}^{2}$ \\
\hline M1 & HCl 0.1 M & 0.0752 & 0.0752 & 0.9994 \\
\hline M8 & Purified water & 0.0294 & 0.0298 & 0.9997 \\
\hline M6 & $\begin{array}{c}\text { Phosphate } \\
\text { buffer pH 7.2 }\end{array}$ & 0.0231 & 0.0236 & 0.9996 \\
\hline M16 & HCl 0.1 M & 0.1072 & 0.1082 & 0.9996 \\
\hline M14 & Purified water & 0.0256 & 0.0254 & 0.9992 \\
\hline M12 & $\begin{array}{c}\text { Phosphate } \\
\text { buffer pH 7.2 }\end{array}$ & 0.0195 & 0.0198 & 0.9974 \\
\hline M22 & HCl 0.1 M & 0.0874 & 0.0878 & 0.9993 \\
\hline M20 & Purified water & 0.0204 & 0.0214 & 0.9970 \\
\hline M27 & $\begin{array}{c}\text { Phosphate } \\
\text { buffer pH 7.2 }\end{array}$ & 0.0254 & 0.0262 & 0.9998 \\
\hline
\end{tabular}

\section{DISCUSSION}

Solubility is one of the most relevant API physicochemical characteristics evaluated in preformulation studies because it is related to drug dissolution and presumably to in vivo performance of the drug product. (17) In early stages of drug discovery, solubility can be evaluated using small amounts of API via intrinsic dissolution testing. (3)

Pyrimethamine is a weak base with pKa 7.4 (15) expected to be ionized at acid $\mathrm{pH}$. This drug had higher observed IDR values for the experiments conducted at $\mathrm{pH} 4.5$ (P4, P5 and P6), followed by pH 1.2 (P1, P2 and P3) and lower values for the experiments P7, P8 and P9, conducted at $\mathrm{pH} 7.2$ (Table 3). Metronidazole is also a weak basic compound and is more soluble at $\mathrm{pH}$ values below 2 (18), which was confirmed by the higher observed IDR values when intrinsic dissolution was performed using $\mathrm{HCl} 0.1$ $M$ as dissolution medium (M1, M16, and M22). Lower observed IDR values for this drug were observed for the experiments at higher $\mathrm{pH}$ test conditions (Table 4).

The determination of IDR from APIs can be used to evaluate and classify the API's solubility according to BCS guidelines when the dose is not too high or very low. (19) Intrinsic dissolution test results show that IDR represents the $\mathrm{pH}$-dependent solubility of pyrimethamine and metronidazole, confirming IDR's suitability to determine the solubility classification of both low and highly soluble drugs. The use of intrinsic dissolution tests to determine the solubility of drugs according to BCS has been reported in the literature. $(3,4)$

For intrinsic dissolution testing, the conditions of surface area, temperature, rotation speed, $\mathrm{pH}$, and ionic strength must be kept constant, as described in Equation 1. $(13,20)$

$$
\left.\frac{d M_{u}}{d t}=-0.62 \gamma A D^{\frac{2}{3}} \omega^{\frac{1}{2}} \mu^{\frac{-1}{6}} C_{s} \quad \text { (Equation } 1\right)
$$

where

$$
\begin{aligned}
& M_{u}=\text { amount of undissolved drug } \\
& \nu=\text { calibration constant (unitless) } \\
& A=\text { surface area of the tablet }\left(\mathrm{cm}^{2}\right) \\
& D=\text { diffusion coefficient }\left(\mathrm{cm}^{2} / \mathrm{min}\right) \\
& \omega=\text { rotational rate of the disk apparatus (radians/sec) } \\
& \mu=\text { dynamic viscosity }\left(\mathrm{g} \mathrm{cm}^{-1} \mathrm{~s}^{-1}\right) \\
& C_{S}=\text { saturation solubility }(\mathrm{mg} / \mathrm{mL})
\end{aligned}
$$

According to Equation 1, IDR increases as the rotation speed is improved, but the statistical analysis for experimental conditions used (independent factors and respective levels) showed that, for pyrimethamine (low soluble drug), the influence of rotation speed was only observed in the experiments conducted using $\mathrm{HCl} \mathrm{pH} 1.2$ (Table 3). For metronidazole, this influence could be noted within the results of the same medium (Table 4), but when comparing to the others, different rotation speeds can lead to similar IDR values. Thus, dissolution medium was the variable that showed significant influence on IDR for pyrimethamine (Figure 1) and metronidazole (Figure 2), with $p<0.05$. 


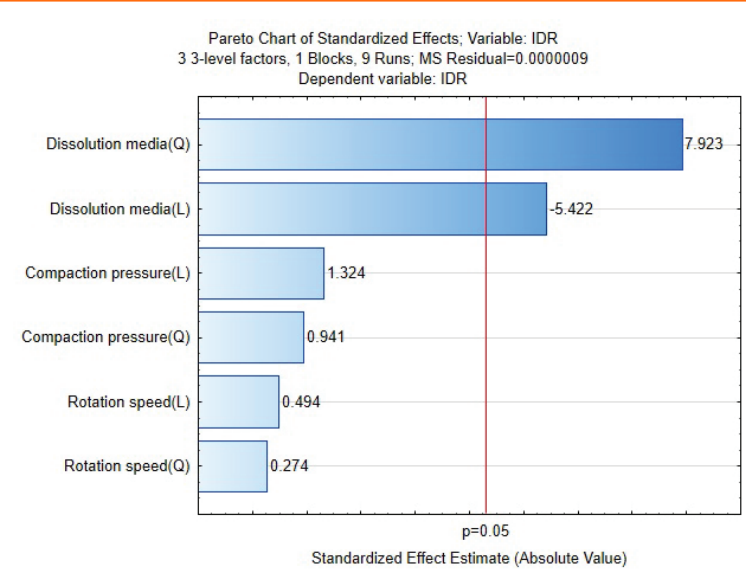

Figure 1. Pareto chart for the effects of variables: dissolution media, compaction pressure, and rotation speed on intrinsic dissolution rate (IDR) for pyrimethamine.

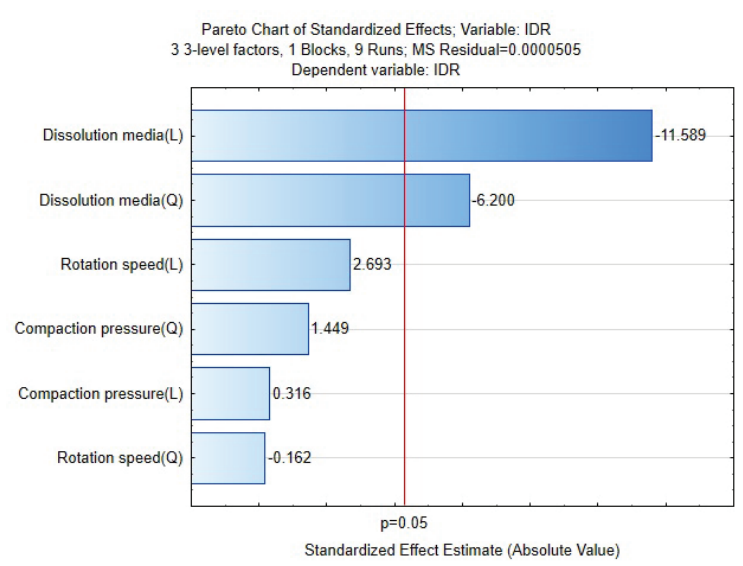

Figure 2. Pareto chart for the effects of variables: dissolution media, compaction pressure, and rotation speed on intrinsic dissolution rate (IDR) for metronidazole.

Due to its higher solubility at $\mathrm{pH} 4.5$, acetate buffer could be chosen as a suitable dissolution medium for intrinsic dissolution tests for pyrimethamine. For metronidazole, although the dissolution medium influenced IDR, all studied media are suitable, due to the high solubility. Any of the tested compaction pressures and rotation speeds for both drugs can be used for the intrinsic dissolution tests, because they did not have a significant effect on IDR; however, it is recommended to fix one set of conditions to compare samples of these drugs from different suppliers.

The simulations obtained in this work showed a high correlation between observed and predicted IDR values for pyrimethamine (Table 3) and metronidazole (Table 4), showing that DDDPlus can be used to estimate the intrinsic dissolution of both, low and highly soluble drugs. The IDR values found for both drugs showed a $\mathrm{pH}$-dependency on their solubility.
DDDPlus was able to predict the intrinsic dissolution values that were used to calculate IDR for each test condition, showing its accuracy in estimating differences in solubility as the $\mathrm{pH}$ changed. IDR values above $0.0017 \mathrm{mg} / \mathrm{s} / \mathrm{cm}^{2}$ indicate a highly soluble drug. $(3,19)$ Pyrimethamine did not show IDR results higher than this value for all $\mathrm{pH}$ tested; therefore, it can be considered a low soluble drug. Metronidazole presented IDR values above $0.0017 \mathrm{mg} / \mathrm{s} /$ $\mathrm{cm}^{2}$, confirming its high solubility. These findings correlate with the BCS classification of the drugs: pyrimethamine class II or IV and metronidazole - class I. (21)

Metronidazole's degree of micronization was used as additional factor by a previous study (11), which confirmed that particle size does not impact IDR. DDDPlus does not use particle size distribution when intrinsic dissolution is selected for simulation, thus micronization differences between metronidazole samples were not considered in simulations. The software was able to simulate IDR values for metronidazole with a high correlation $R^{2} \geq 0.9970$ (Table 4) with the in vitro data for different intrinsic dissolution test conditions.

DDDPlus can accurately predict IDR for the studied drugs, showing that this software can be used to estimate drug's solubility according to BCS classification and the best conditions for intrinsic dissolution tests, reducing the number of laboratory experiments, and helping pharmaceutical companies to save time and costs.

\section{CONCLUSION}

Computer simulations using DDDPlus can help to gain biopharmaceutical understanding of APIs in the drug development process. Software simulations can be used to predict the intrinsic dissolution of API's in the physiologically relevant $\mathrm{pH}$ range of 1 to 7.2. This can help streamline and minimize experimental lab work. Key experiments can be identified by the simulations and be confirmed by lab results to characterize important biopharmaceutical API properties.

\section{ACKNOWLEDGEMENTS}

The authors would like to thank Simulations Plus for having provided the software and National Council of Scientific and Technological Development (CNPq/Brazil) for the grant of Institutional Scholarship Program for Scientific Initiation (PIBIC/CNPq/Brazil) and (CNPq/Brazil) (400455/2014-5).

\section{CONFLICT OF INTEREST}

No conflict of interest has been declared by the authors. 


\section{REFERENCES}

1. <1087> Apparent intrinsic dissolution - dissolution testing procedures for rotating disk and stationary disk. In The United States Pharmacopeia and National Formulary USP 38-NF 33; The United States Pharmacopeial Convention, Inc.: Rockville, MD, 2015.

2. Wang, J.; Flanagan D. R. Fundamentals of dissolution. In: Developing Solid Oral Dosage Forms: Pharmaceutical Theory and Practice; Qiu, Y.; Chen, Y.; Zhang, G. G. Z.; Liu, L.; Porter, W. R., Eds.; Academic Press: New York, 2009; pp 309-313.

3. Issa, M. G.; Ferraz, H. G. Intrinsic dissolution as a tool for evaluating drug solubility in accordance with the Biopharmaceutics Classification System. Dissolution Technol. 2011, 18 (3), 6-11. DOI: dx.doi.org/10.14227/DT180311P6.

4. Dezani, A. B.; Pereira, T. M.; Caffaro, A. M.; Reis, J. M.; Serra, C. H. R. Equilibrium solubility versus intrinsic dissolution: characterization of lamivudine, stavudine and zidovudine for BCS classification. Braz J Pharm Sci. 2013, 49 (4), 853-863. DOI: http://dx.doi.org/10.1590/S1984-82502013000400026.

5. Amidon, G. L.; Lennernäs, H.; Shah, V. P.; Crison, J. R. A theoretical basis for a biopharmaceutic drug classification: the correlation of in vitro drug product dissolution and in vivo bioavailability. Pharm. Res. 1995, 12 (3), 413-420. DOI: 10.1023/A:1016212804288.

6. Guidance for industry: Waiver of in vivo bioavailability and bioequivalence studies for immediate-release solid oral dosage forms based on a biopharmaceutics classification system. U.S. Department of Health and Human Services, Food and Drug Administration, Center for Drug Evaluation and Research (CDER), U.S. Government Printing Office: Washington, DC, 2000.

7. Draft Guidance for industry: Waiver of in vivo bioavailability and bioequivalence studies for immediate-release solid oral dosage forms based on a biopharmaceutics classification system. U.S. Department of Health and Human Services, Food and Drug Administration, Center for Drug Evaluation and Research (CDER), U.S. Government Printing Office: Washington, DC, 2015.

8. Breda, S. A.; Jimenez-Kairuz, A. F.; Manzo, R. H.; Oliveira, M. E. Solubility behavior and biopharmaceutical classification of novel high-solubility ciprofloxacin and norfloxacin pharmaceutical derivatives. Int. J. Pharm. 2009, 371 (1-2), 106-113. DOI: 10.1016/j.ijpharm.2008.12.026.

9. Zhang, J.; Liu, D.; Huang, Y.; Gao, Y.; Qian, S. Biopharmaceutics classification and intestinal absorption study of apigenin. Int. J. Pharm. 2012, 436 (1-2), 311-317. DOI: 10.1016/j. ijpharm.2012.07.002.

10. Ono, A.; Sugano, K. Application of the BCS biowaiver approach to assessing bioequivalence of orally disintegrating tablets with immediate release formulations. Eur. J. Pharm. Sci. 2014, 64, 37-43. DOI: 10.1016/j.ejps.2014.08.003.

11. Issa, M. G.; Duque, M. D.; Souza, F. M.; Ferraz, H. G. Evaluating the impact of different variables in the intrinsic dissolution of metronidazole. Int. J. Pharm. Engineering 2013, 1, 17-29.

12. Giorgetti, L.; Issa, M. G.; Ferraz, H. G. The effect of dissolution medium rotation speed and compaction pressure on the intrinsic dissolution rate of amlodipine besylate, using the rotating disk method. Braz. J. Pharm. Sci. 2014, 50 (3), 513-520. DOI: 10.1590/S1984-82502014000300009.

13. Simulations Plus, DDDPlus ${ }^{\mathrm{TM}}$ version 4.0 Manual, California, USA, 2011.

14. Almukainzi, M.; Okumu, A.; Wei, H.; Löbenberg, R. Simulation of in vitro dissolution behavior using DDDPlus ${ }^{\mathrm{TM}}$. AAPS PharmSci. 2015, 16 (1), 217-221. DOI: 10.1208/s12249-014-0241-5.

15. Amin, N. C.; Blanchin, M-D, Aké, M.; Fabre H. Capillary zone electrophoresis as a potential technique for the simultaneous determination of sulfadoxine and pyrimethamine in tablet formulations. J. Pharm. Biom. Anal. 2012, 58, 168-171. DOI: 10.1016/j.jpba.2011.09.028.

16. Shemer, H.; Kunukcu, Y. K.; Linden, K. G. Degradation of the pharmaceutical metronidazole via UV, Fenton and photo-Fenton processes. Chemosphere. 2006, 63 (2), 269-276. DOI: 10.1016/j. chemosphere.2005.07.029.

17. Borhade, V.; Pathak, S.; Sharma, S.; Patravale, V. Clotrimazole nanoemulsion for malaria chemotherapy. Part I: preformulation studies, formulation design and physicochemical evaluation. Int. J. Pharm. 2012, 431 (1-2), 138-148. DOI: 10.1016/j. ijpharm.2011.12.040.

18. Wu, Y.; Fassihi R. Stability of metronidazole, tetracycline $\mathrm{HCl}$ and famotidine alone and in combination. Int. J. Pharm. 2005, 290 (1-2), 1-13. DOI: 10.1016/j.ijpharm.2004.10.015.

19. Yu, L. X.; Carlin, A. S.; Amidon, G. L.; Hussain, A. S. Feasibility studies of utilizing disk intrinsic dissolution rate to classify drugs. Int. J. Pharm. 2004, 270 (1-2), 221-227. DOI: 10.1016/j. ijpharm.2003.10.016.

20. Mauger, J.; Ballard, J.; Brockson, R.; De, S.; Gray, V.; Robinson, D. Intrinsic dissolution performance testing of the USP dissolution apparatus 2 (rotating paddle) using modified salicylic acid calibrator tablets: proof of principle. Dissolution Technol. 2003, 10 (3), 6-15. DOI: dx.doi.org/10.14227/DT100303P6.

21. Lindenberg, M.; Kopp, S.; Dressman J. B. Classification of orally administered drugs on the World Health Organization Model List of Essential Medicines according to the biopharmaceutics classification system. Eur. J. Pharm. Biopharm. 2004, 58 (2), 265278. DOI: 10.1016/j.ejpb.2004.03.001. 